\title{
A New Differential Approach for Parametric-Implicit Surface Intersection
}

\author{
J. Puig-Pey, A. Gálvez, and A. Iglesias \\ Department of Applied Mathematics and Computational Sciences, University of \\ Cantabria, Avda. de los Castros, s/n, E-39005, Santander, Spain \\ \{puigpeyj, galveza, iglesias\}@unican.es
}

\begin{abstract}
In this paper, we focus on the parametric-implicit surface intersection problem. In our approach, this problem is formulated in terms of an initial value problem of first-order ordinary differential equations (ODEs). To this end, we take advantage of the orthogonality at any point on the intersection curve between the tangent vector to that curve and the normal vector to the implicit surface. This yields an initial value system of ODEs that is numerically integrated through an adaptive Runge-Kutta method. In order to determine the initial value for this system, a simple procedure based on the scalar and vector fields associated with the function defining the implicit surface and its gradient is described. Such a procedure yields a starting point on the nearest branch of the intersection curve. The performance of the presented method is analyzed by means of some illustrative examples.
\end{abstract}

\section{Introduction}

The intersection of surfaces is one of the most outstanding problems in many fields, such as computational geometry, solid modeling, geometric processing, visualization, analysis, and manufacturing of 3D entities. It appears in the countouring of surfaces [10] (intersection with series of parallel planes, cylinders, cones, etc), in numerical-controlled machining [6] (intersection of offset surfaces with series of parallel planes), in the boundary (B-rep) representation for Constructive Solid Geometry models of the objects, in manufacturing [4] (slicing operations for rapid prototyping, determination of collisions), etc.

During the last few years, a number of different methods to compute the intersection of surfaces have been described in the literature1. Basically, they can be classified into analytical and numerical methods. Analytical methods seek exact solutions by finding some function describing the intersection curves. Although unaffected by robustness and efficiency limitations, they require many different algorithms designed ad hoc for each kind of surface involved. Furthermore, they cannot deal with non-algebraic surfaces and hence numerical methods are

\footnotetext{
${ }^{1}$ See, for example, the excellent reviews on this topic in [11 (Chapter 12) or [16] (Chapter 5) and the references therein. We also recommend the survey in [19]. Additional references can be found in 7 .
} 
usually applied instead. At their turn, numerical methods can be classified into four main categories: lattice evaluation, recursive subdivision, marching schemes and continuation methods 19]. Another alternative classification of the surface intersection methods is given by the kind of surfaces involved. For instance, Patrikalakis and Maekawa 16] distinguish four different kinds of surfaces for intersection: rational polynomial parametric (RPP), procedural parametric ( $\mathrm{PP})$, implicit algebraic (IA) and implicit procedural (IP) (see Chapter 5 of [16]).

In this paper, we address the parametric-implicit surface intersection problem, that is, the problem of computing the intersection of a parametric surface 2 $\mathbf{S}(u, v)$ and an implicit surface $f(x, y, z)=0$ (this obviously includes the case of the RPP-IA surface intersection in Patrikalakis-Maekawa's terminology). This problem has a great interest for numerically controlled machining (milling) to create machining paths for the cutting tools. In this task, we must assure that the tool does not collide with the surface being milled at some undesired point. As pointed out by several authors [6]11], while the surfaces to be milled are frequently free-form rational parametric surfaces, namely NURBS, the most commonly used cutting tools have cylindrical (flat-endmill), spherical (ball-endmill) or toroidal (round-endmill) shapes, which can be faithfully represented by implicit surfaces.

A number of different solutions for this problem have been suggested. Most of them [11|16] are based on the idea of inserting the equations of the parametric surface into the equation of the implicit one to get such intersection curve. For low degree implicit surfaces it is possible often to obtain an implicit curve in the parametric domain $(u, v) \quad 1519$ but it still remains a problem for high degree surfaces. Other proposals include a combination of algebraic and analytical methods [8], hybrid algorithms combining subdivision (based on the divide-and-conquer methodologies), tracing and numerical methods (mainly Newton's method) [13, etc. Unfortunately, they exhibit a substantial loss of accuracy making them unsuitable for practical applications. Finally, there is a family of methods known as marching methods based on generating a sequence of points of an intersection curve branch by stepping from a point on such a curve in a direction determined by some local differential geometry analysis [2, 314 . These methods are globally incomplete since they require starting points for every branch of the solution. Motivated by this, great effort has been devoted to the determination of such starting points by using hodographs [20], elimination methods [5] or by two local methods, namely iterative optimization and Moore-Penrose pseudo-inverse method [1].

Another approach that is gaining interest during the last few years is the differential scheme such as the second order boundary algebraic-differential approach in 9 10]. The method presented in this paper belongs to this last family of methods. In our approach, the parametric-implicit surface intersection problem is formulated in terms of an initial value problem of first-order ordinary differential equations (ODEs). For doing so, we take advantage of the orthogonality at any point on the intersection curve between the tangent vector to

\footnotetext{
${ }^{2}$ In this paper vectors will be denoted in bold.
} 
that curve and the normal vector to the implicit surface. The resulting initial value system of ODEs is then numerically integrated through an adaptive fourthorder Runge-Kutta method [18]. From this point of view, the present method can also be classified as a marching method. As remarked above, one of the main shortcomings of the marching methods is the need of a starting point on the intersection curve. In this paper, a procedure to overcome this limitation is proposed. Starting at an arbitrary point on the parametric surface, we trace a path on that surface by following the direction indicated by the gradient of the implicit surface. As we will show later, this simple procedure yields a starting point on the nearest branch of the intersection curve. The performance of this procedure will be shown by means of some illustrative examples.

The structure of this paper is as follows. In Section 2 we introduce some basic concepts and and the terminology to be used throughout the paper. Then a new method for computing the intersection between two surfaces given in parametric and implicit forms is presented in Section 3 . The determination of a starting point for the marching method of this paper is discussed in Section 4 while Section 5 gives some brief comments about the numerical procedures. In Section 6] some illustrative examples to show the good performance of the proposed method are presented. Finally, the conclusions and future work are discussed in Section 7

\section{Basic Concepts and Terminology}

In this paper we restrict ourselves to both parametric and implicit surfaces. In addition, it is assumed that they are also differentiable at any point. The parametric surfaces are described by a vector-valued function of two variables:

$$
\mathbf{S}(u, v)=(x(u, v), y(u, v), z(u, v)), \quad u, v \in \Omega \subset \mathbb{R}^{2}
$$

where $u$ and $v$ are the surface parameters. Expression (1) is called a parameterization of the surface $\mathbf{S}$. At regular points, the partial derivatives $\mathbf{S}_{u}(u, v)$ and $\mathbf{S}_{v}(u, v)$ do not vanish simultaneously. These partial derivatives define the unit normal vector $\mathbf{N}$ to the surface at $\mathbf{S}\left(u_{0}, v_{0}\right)$ as:

$$
\mathbf{N}=\frac{\mathbf{S}_{u} \times \mathbf{S}_{v}}{\left|\mathbf{S}_{u} \times \mathbf{S}_{v}\right|}
$$

where " $\times$ " denotes the cross product.

A curve in the domain $\Omega$ can be described by means of its parametric representation $\{u=u(t), v=v(t)\}$. This expression defines a three-dimensional curve $\mathbf{C}(t)$ on the surface $\mathbf{S}$ given by $\mathbf{C}(t)=\mathbf{S}(u(t), v(t))$. Applying the chain rule, the tangent vector $\mathbf{C}^{\prime}(t)$ of this curve at a point $\mathbf{C}(t)$ becomes:

$$
\mathbf{C}^{\prime}(t)=\mathbf{S}_{u} u^{\prime}(t)+\mathbf{S}_{v} v^{\prime}(t)
$$

In this work the curve $\mathbf{C}$ will usually be parameterized by the arc-length $s$ on the surface. Its geometric interpretation is that a constant step $s$ traces a constant distance along an arc-length parameterized curve. Since some industrial 
operations require an uniform parameterization, this property has several practical applications. For example, in computer controlled milling operations, the curve path followed by the milling machine must be parameterized such that the cutter neither speeds up nor slows down along the path. Consequently, the optimal path is that parameterized by the arc-length. In this case:

$$
E\left(\frac{d u}{d s}\right)^{2}+2 F \frac{d u}{d s} \frac{d v}{d s}+G\left(\frac{d v}{d s}\right)^{2}=1
$$

where $E, F$ and $G$ are the coefficients of the First Fundamental Form of the surface given by:

$$
E=\mathbf{S}_{u} \cdot \mathbf{S}_{u} \quad, \quad F=\mathbf{S}_{u} \cdot \mathbf{S}_{v} \quad, \quad G=\mathbf{S}_{v} \cdot \mathbf{S}_{v}
$$

and "." indicates the dot product (see [21] for details). For the sake of clarity, in this paper the parameter $s$ will be strictly used to refer to a curve parameterized by the arc-length on the surface.

Another useful tool we will use in Section 4 is given by those curves which are tangent to the projection on a surface of a given vector field $\mathbf{D}(x, y, z)$ defined in $\mathbb{R}^{3}$. For example, for the constant vector $\mathbf{D}=(0,0,1)$ the curves generated by this procedure on a surface representing a terrain are actually the trajectories followed by the water droplets on such a terrain under the effect of gravity.

As shown in [12], these curves can easily be described through a system of ODEs. Suppose that we are given an arbitrary point $\mathbf{P}=(x, y, z)$ on the surface $\mathbf{S}(u, v)$, the normal vector $\mathbf{N}$ to the surface at $\mathbf{P}$ and the vector value $\mathbf{D}$ at $\mathbf{P}$ of a known vector field $\mathbf{D}(x, y, z)$. We look for the curve $\mathbf{C}(s)$ on $\mathbf{S}$ which is tangent to the orthogonal projection of $\mathbf{D}$ onto the tangent plane to $\mathbf{S}$ at $\mathbf{P}$.

It is obvious that $\mathbf{C}^{\prime}(s)$ is on the tangent plane to $\mathbf{S}$ at $\mathbf{P}$. The differential equation for the curve is obtained from the orthogonality of the tangent vector $\mathbf{C}^{\prime}(s)$ and the vector $\mathbf{D} \times \mathbf{N}$, i.e., $\mathbf{C}^{\prime}(s) .(\mathbf{D} \times \mathbf{N})=0$. From (3) we get:

$$
\mathbf{S}_{u} .(\mathbf{D} \times \mathbf{N}) \frac{d u}{d s}+\mathbf{S}_{v} \cdot(\mathbf{D} \times \mathbf{N}) \frac{d v}{d s}=0
$$

Combining (4) and (6), the following first-order explicit autonomous system of ODEs for $\frac{d u}{d s}$ and $\frac{d v}{d s}$ is obtained:

$$
\left\{\begin{array}{l}
\frac{d u}{d s}= \pm \frac{\mathbf{S}_{v} \cdot(\mathbf{D} \times \mathbf{N})}{\sqrt{E\left(\mathbf{S}_{v} \cdot(\mathbf{D} \times \mathbf{N})\right)^{2}-2 F\left(\mathbf{S}_{u} \cdot(\mathbf{D} \times \mathbf{N})\right)\left(\mathbf{S}_{v} \cdot(\mathbf{D} \times \mathbf{N})\right)+G\left(\mathbf{S}_{u} \cdot(\mathbf{D} \times \mathbf{N})\right)^{2}}} \\
\frac{d v}{d s}=\mp \frac{\mathbf{S}_{u} \cdot(\mathbf{D} \times \mathbf{N})}{\sqrt{E\left(\mathbf{S}_{v} \cdot(\mathbf{D} \times \mathbf{N})\right)^{2}-2 F\left(\mathbf{S}_{u} \cdot(\mathbf{D} \times \mathbf{N})\right)\left(\mathbf{S}_{v} \cdot(\mathbf{D} \times \mathbf{N})\right)+G\left(\mathbf{S}_{u} \cdot(\mathbf{D} \times \mathbf{N})\right)^{2}}}
\end{array}\right.
$$

with the initial conditions

$$
\left\{\begin{array}{l}
u(0)=u_{0} \\
v(0)=v_{0}
\end{array}\right.
$$

which correspond to an initial point $\mathbf{P}_{0}=\left(x_{0}, y_{0}, z_{0}\right)$ on the curve $\mathbf{C}$. 
On the other hand, an implicit surface is given by $f(x, y, z)=0$ where $f$ is a differentiable scalar function. In this case, the unit normal vector $\mathbf{N}$ to the surface at a non-singular point $\mathbf{P}_{0}=\left(x_{0}, y_{0}, z_{0}\right)$ is $\mathbf{N}=\frac{\left(f_{x}, f_{y}, f_{z}\right)}{\left\|\left(f_{x}, f_{y}, f_{z}\right)\right\|_{2}}$ evaluated at $\mathbf{P}_{0}$.

\section{The Parametric-Implicit Surface Intersection Method}

Let us consider two surfaces $S_{1}$ and $S_{2}$ given in parametric form $\mathbf{S}(u, v)$ and in implicit form $f(x, y, z)=0$, respectively. Let $\mathbf{N}_{2}=\left(f_{x}, f_{y}, f_{z}\right)$ be the unit normal vector to $S_{2}$ at a point on the intersection curve $\mathbf{C}(s)$ between $S_{1}$ and $S_{2}$, with $s$ the arc-length on $S_{1}$. Following (3), the unit tangent vector $\mathbf{T}_{1}(s)$ to the curve $\mathbf{C}(s)$ considered as belonging to $S_{1}$ is given by:

$$
\mathbf{T}_{1}(s)=\frac{d \mathbf{C}(s)}{d s}=\mathbf{S}_{u} \frac{d u}{d s}+\mathbf{S}_{v} \frac{d v}{d s}
$$

Because $\mathbf{T}_{1}$ and $\mathbf{N}_{2}$ are orthogonal

$$
\mathbf{T}_{1}(s) \cdot \mathbf{N}_{2}=0 \Longrightarrow\left(\mathbf{S}_{u} \cdot \mathbf{N}_{2}\right) \frac{d u}{d s}+\left(\mathbf{S}_{v} \cdot \mathbf{N}_{2}\right) \frac{d v}{d s}=0
$$

Solving (4) and (9) for $\frac{d u}{d s}$ and $\frac{d v}{d s}$, we obtain:

$$
\left\{\begin{array}{l}
\frac{d u}{d s}= \pm \frac{\mathbf{S}_{v} \cdot \mathbf{N}_{2}}{\sqrt{E\left(\mathbf{S}_{v} \cdot \mathbf{N}_{2}\right)^{2}-2 F\left(\mathbf{S}_{v} \cdot \mathbf{N}_{2}\right)\left(\mathbf{S}_{u} \cdot \mathbf{N}_{2}\right)+G\left(\mathbf{S}_{u} \cdot \mathbf{N}_{2}\right)^{2}}} \\
\frac{d v}{d s}=\mp \frac{\mathbf{S}_{u} \cdot \mathbf{N}_{2}}{\sqrt{E\left(\mathbf{S}_{v} \cdot \mathbf{N}_{2}\right)^{2}-2 F\left(\mathbf{S}_{v} \cdot \mathbf{N}_{2}\right)\left(\mathbf{S}_{u} \cdot \mathbf{N}_{2}\right)+G\left(\mathbf{S}_{u} \cdot \mathbf{N}_{2}\right)^{2}}}
\end{array}\right.
$$

which together with an initial point of the intersection curve:

$$
\left\{\begin{array}{l}
u(0)=u_{0} \\
v(0)=v_{0}
\end{array}\right.
$$

constitutes an initial value problem for this system of explicit first-order ordinary differential equations. The signs \pm and $\mp$ in $(10)$ mean that there are two arcs of curve starting at $\left(u_{0}, v_{0}\right)$ associated with the two possible opposite directions of the tangent vector $\mathbf{T}_{1}(s)$. Note also that the vector $\mathbf{N}_{2}$ can be evaluated in the expressions above because coordinates $(x, y, z)$ are calculated through the equations of $S_{1}, \mathbf{S}(u, v)$.

\section{Starting Point of Intersection Curve between Two Surfaces}

In the previous section an initial value system of ODEs associated with an intersection curve between a parametric surface and an implicit one has been 
Table 1. Algorithm for the determination of the starting point

Input: $\quad$ a parametric surface $S_{1}$ given by $\mathbf{S}(u, v)$ an implicit surface $S_{2}$ given by $f(x, y, z)=0$ an arbitrary point $\mathbf{P}_{0}$ on $S_{1}$

Output: $\quad$ a starting point $\mathbf{P}^{*}$ on the intersection curve between $S_{1}$ and $S_{2}$

Procedure: if $f\left(\mathbf{P}_{0}\right)>0$, then set $k=-1$ else set $k=1$

Integrate system (7)-(8) with $\mathbf{D}=k . \nabla f\left(\mathbf{P}_{i}\right)$ until we reach a point

$\mathbf{P}^{*}$ at which $f\left(\mathbf{P}^{*}\right)=0$

determined. For such a system to be unambiguously defined, we must provide a starting point on that intersection curve. As remarked in Section 1, several methods have been described to solve this problem. In this section a new approach to determine such a starting point is discussed. The basic idea behind this method is to take advantage of the scalar and vector fields associated with the function of the implicit surface $f(x, y, z)=0$ and its gradient.

On one hand, the function $f(x, y, z)$ defining the implicit surface can be seen as a scalar field splitting up $\mathbb{R}^{3}$ into two disjoint regions, namely $f(x, y, z)>0$ and $f(x, y, z)<0$, respectively. Any continuous path from one region to the another one must necessarily pass through the implicit surface. The corresponding path-surface intersection point can easily be obtained by simply checking for a change of the sign of the scalar value $f(x, y, z)$. This could be considered as a "curved ray tracing" method for point location on the implicit surface. On the other hand, the gradient of $f(x, y, z)$, given by $\nabla(f)=\left(f_{x}, f_{y}, f_{z}\right)$, can be considered as a vector field in $\mathbb{R}^{3}$ as follows: for any three-dimensional point $\mathbf{P}=$ $(x, y, z)$, its corresponding vector value $\mathbf{D}$ is given by $\mathbf{D}(x, y, z)=\nabla(f(x, y, z))$.

These scalar and vector fields can be successfully applied to the determination of a starting point on the intersection curve between the parametric and the implicit surfaces. The basic idea can be summarized as follows: firstly we consider an arbitrary point on the parametric surface. Then, we move on that surface from this initial point following one of the directions of the gradient vector field $\pm \nabla f= \pm\left(f_{x}, f_{y}, f_{z}\right)$ where $f$ is the function defining the implicit surface. The corresponding trajectory is obtained by numerical integration of the system (7)-(8) (see Section 22). This numerical integration is accomplished until we reach a point on the implicit surface (i.e., a point at which $f(x, y, z)=0$ ). The corresponding algorithm is shown in Table 1.

\section{Numerical Integration of the ODEs}

The calculation of the analytical expression for $u$ and $v$ from (10) is not possible in general. Fortunately, the systems (77)-(18) and (10)-(11) only involve first-order 
explicit ODEs and hence many standard numerical techniques can be applied instead. In particular, all the numerical work has been performed by using an adaptive 4-5-order Runge-Kutta algorithm [18] with control of absolute and relative error tolerance. In all our examples, the absolute tolerance error (a threshold below which the value of the $i$ th solution component is unimportant) was $10^{-7}$ while the relative tolerance error (a measure of the error relative to the size of each solution component) was $10^{-4}$.

In general, the method works well for any couple of parametric-implicit surfaces. It should be noticed, however, that some situations require a careful analysis: for example, when the piece consists of several parametric surfaces some kind of continuity conditions must be imposed to assure that the differential model is still valid in the neighbourhood of each surface boundary. On the other hand, the domain of the involved surfaces should be taken into account during the integration process. For example, the integration of (17)-(8) to find a point on the intersection curve will stop if:

- the trajectory reaches a singular point (for example, if either $\mathbf{N}$ or $\mathbf{D}$ in (7) vanishes),

- $\mathbf{N}$ and $\mathbf{D}$ are parallel, corresponding to a maximum, minimum or saddle point on the surface with respect to the direction of vector field $\mathbf{D}$,

- the trajectory reaches the limits of the domain of the parametric surface or

- the trajectory reaches a zero value for $f(x, y, z)$, meaning that such a point on the intersection curve has been obtained.

Note that such an integration yields a starting point on the nearest branch of the intersection curve. Therefore, in the case of working with surfaces with several branches or with trimmed surfaces (to quote just two examples), additional tests for self-intersection and searching of additional branches must be performed. Once a starting point is obtained, the system (10)-11 to obtain the intersection curve between the two surfaces is integrated with the starting point as initial value.

\section{$6 \quad$ Some Illustrative Examples}

In general, we can choose any parametric surface to be interesected with an implicit one. However, because of their advantages in industrial environments, their flexibility and the fact that they can represent well a wide variety of shapes, in this paper we will focus on NURBS surfaces. In this case, the corresponding derivatives needed in (7) and (10) have been obtained as described in [17.

As a first example, Fig. 1(left) shows the intersection between a $(4,4)$-order NURBS surface defined by a grid of $4 \times 4$ control points and nonperiodic knot vectors for both $u$ and $v$ and the "quartic cylinder" surface, an implicit surface given by $\left(x^{2}+y^{2}\right) z^{2}+\left(\frac{1}{2}\right)^{2}\left(x^{2}+y^{2}-1\right)=0$. In this picture, an star indicates the arbitrary point on the NURBS surface at which the algorithm described in Table 1 is applied. As described in Section 4 this algorithm yields a path 

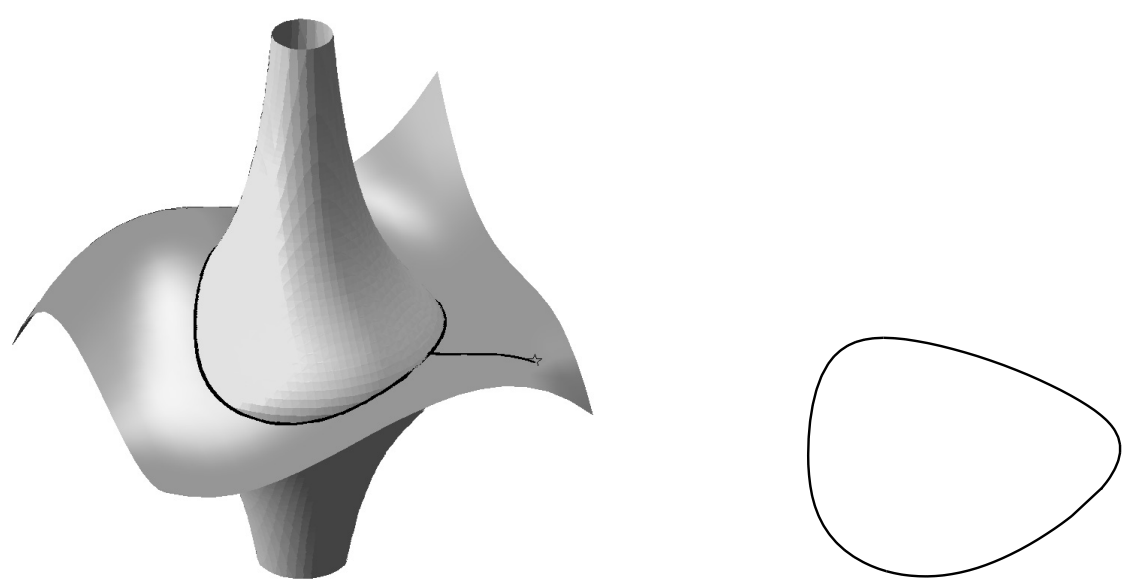

Fig. 1. (left) Intersection of a $(4,4)$-order nonperiodic NURBS surface and the implicit surface $\left(x^{2}+y^{2}\right) z^{2}+\left(\frac{1}{2}\right)^{2}\left(x^{2}+y^{2}-1\right)=0$; (right) intersection curve
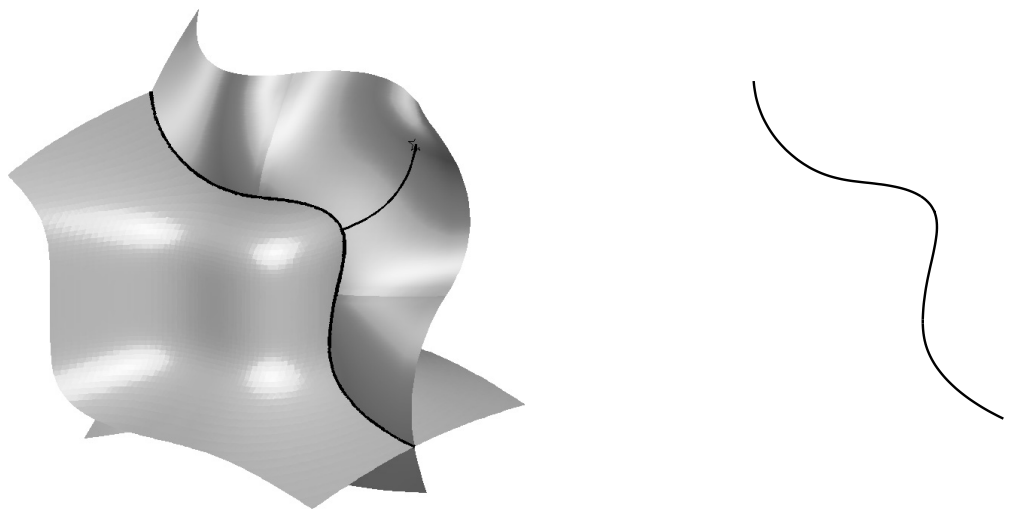

Fig. 2. (left) Intersection of a $(3,3)$-order nonperiodic NURBS surface and the implicit surface $x^{2}+y^{3}+z^{5}-1=0$; (right) intersection curve

on the parametric surface from this arbitrary point to a starting point on the intersection curve between both surfaces. As shown in this figure, this algorithm exhibits a very good performance. This is not surprising since we impose the path to follow the direction determined by the projection of the gradient of $f(x, y, z)$ on the parametric surface. This curve is displayed as a continuous line in both Figs. T(left) and (right).

Figure 2 shows the intersection between a $(3,3)$-order nonperiodic NURBS surface defined by a grid of $4 \times 4$ control points and the implicit surface given by $x^{2}+y^{3}+z^{5}-1=0$ (left) as well as the obtained intersection curve (right). 

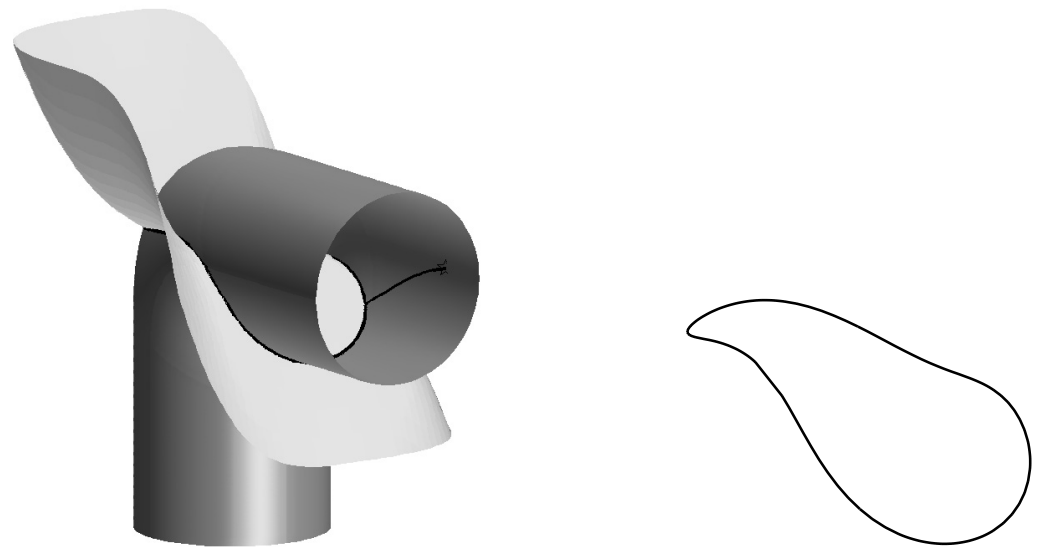

Fig. 3. (left) Intersection of a (3,3)-order nonuniform NURBS surface and the implicit surface $\left(\frac{x}{2}\right)^{3}-\left(\frac{y}{2}\right)^{3}-\frac{z}{2}=0 ;$ (right) intersection curve

Note that the parametric surface consists of two patches in both the $u$ and $v$ directions. Finally, Figure 3 displays the intersection between a $(3,3)$-order nonuniform NURBS surface defined by a grid of $9 \times 5$ control points with knot vectors $\mathcal{U}=\left[0,0,0, \frac{1}{3}, \frac{2}{3}, 1,1,1\right]$ and $\mathcal{V}=\left[0,0,0, \frac{1}{4}, \frac{1}{4}, \frac{2}{4}, \frac{2}{4}, \frac{3}{4}, \frac{3}{4}, 1,1,1\right]$ for the $u$ and $v$ directions respectively and the "cubic saddle" surface, an implicit surface given by $\left(\frac{x}{2}\right)^{3}-\left(\frac{y}{2}\right)^{3}-\frac{z}{2}=0$.

\section{Conclusions and Future Work}

In this paper, a new method for solving the parametric-implicit surface intersection problem is proposed. In our approach, this problem is formulated in terms of an initial value problem of first-order ordinary differential equations (ODEs), which is numerically integrated through an adaptive 4-5-order RungeKutta method. In order to determine the initial value for this system, a simple procedure based on the scalar and vector fields associated with the function defining the implicit surface and its gradient is described. Such a procedure yields a starting point on the nearest branch of the intersection curve. The method is very general and it can be applied to any couple of implicit-parametric surfaces.

Although the method has shown a very good performance in the examples described in this paper, there is still a long way to walk. Future improvements include the cases of self-intersecting surfaces and the analysis of the stability of this method for singular points. On the other hand, the determination of different branches for the intersection curves is still an open problem that requires further research. These and other open questions are currently under analysis and the future results will be reported elsewhere. 
Acknowledgments. The authors are grateful to the CICYT of the Spanish Ministry of Science and Technology (project DPI2001-1288) and the University of Cantabria for partial support of this work.

\section{References}

1. Abdel-Malek, K. Yeh, H.-J.: On the determination of starting points for parametric surface intersections, Computer-Aided Design 28(1) (1997) 21-35

2. Bajaj, C., Hoffmann, C. M., Hopcroft, J. E. H., Lynch, R. E.: Tracing surface intersections, Computer Aided Geometric Design 5 (1988) 285-307

3. Barnhill, R.E., Kersey, S.N.: A marching method for parametric surface/surface intersection, Computer Aided Geometric Design 7 (1990) 257-280

4. Barnhill, R.E. (Ed.): Geometry Procesing for Design and Manufacturing, SIAM, Philadelphia (1992)

5. Chandru, V., Dutta, D, Hoffmann, C.M.: On the geometry of Dupin cyclides, The Visual Computer 5 (1989) 277-290

6. Choi, B.K., Jerard, R.B: Sculptured Surface Machining. Theory and Applications. Kluwer Academic Publishers, Dordrecht/Boston/London (1998)

7. Farin, G.: An SSI Bibliography. In: Barnhill, R. (ed.) Geometry Processing for Design and Manufacturing, SIAM (1992) 205-207

8. Farouki, R.T.: Direct surface section evaluation. In: Farin, G. (ed.), Geometric Modeling. Algorithms and New Trends, SIAM (1987) 319-334

9. Grandine, T.A., Klein, F.W.: A new approach to the surface intersection problem, CAGD 14 (1997) 111-134

10. Grandine, T.A.: Applications of contouring, SIAM Review 42 (2000) 297-316

11. Hoschek, J., Lasser, D.: Computer-Aided Geometric Design, A.K. Peters, Wellesley, MA (1993)

12. Iglesias, A., Gálvez, A., Puig-Pey, J.: Generating drop trajectories on parametric surfaces. In: Peng, Q., Li, W., Yu, J. (Eds.): Proceedings of the Seventh CAD/ Graphics'2001, International Academic Publishers/World Publishing Corporation, Beijing (2001) 350-357

13. Kriezis, G.A., Prakash, P.V., Patrikalakis, N.M.: Method for intersecting algebraic surfaces with rational polynomial patches, Computer-Aided Design 22(10) (1990) 645-654

14. Kriezis, G.A., Patrikalakis, N.M., Wolters, F.-E.: Topological and differentialequation methods for surface reconstructions, Computer-Aided Design 24(1) (1992) 41-55

15. Krishnan, S, Manocha, D.: Efficient surface intersection algorithm based on lowerdimensional formulation, ACM Transaction on Graphics 16(1) (1997) 74-106

16. Patrikalakis, N.M., Maekawa, T.: Shape Interrogation for Computer Aided Design and Manufacturing. Springer-Verlag New York Berlin Heidelberg (2002)

17. Piegl, L., Tiller, W.: The NURBS Book, Springer Verlag, Berlin Heidelberg (1997)

18. Press, W.H., Teukolsky, S.A., Vetterling, W.T., Flannery, B.P.: Numerical Recipes (2nd edition), Cambridge University Press, Cambridge (1992)

19. Pratt, M.J., Geisow, A.D.: Surface-surface intersection problems. In: Gregory, J.A. (ed.) The Mathematics of Surfaces, Clarendon Press, Oxford (1986) 117-142

20. Sederberg, T.W., Meyers, R.J.: Loop detection in surface patch intersections, Computer Aided Geometric Design 5 (1988) 161-171

21. Struik, D.J.: Lectures on Classical Differential Geometry, 2nd ed., Dover Publications, New York (1988) 